\title{
Maximally Rugged NK Landscapes Contain the Highest Peaks
}

\author{
Benjamin Skellett ${ }^{1}$ \\ ben@itee.uq.edu.au
}

\author{
Benjamin Cairns ${ }^{2}$ \\ bjc@maths.uq.edu.au
}

\author{
Nicholas Geard ${ }^{1}$ \\ nic@itee.uq.edu.au
}

\author{
Bradley Tonkes ${ }^{4}$ \\ btonkes@cse.unsw.edu.au j.wiles@itee.uq.edu.au \\ ${ }^{1}$ School of Information Technology and Electrical Engineering \\ ${ }^{2}$ Department of Mathematics \\ ${ }^{3}$ School of Psychology \\ The University of Queensland, Brisbane, Australia \\ ${ }^{4}$ School of Computer Science and Engineering \\ The University of New South Wales, Sydney, Australia
}

\begin{abstract}
NK models provide a family of tunably rugged fitness landscapes used in a wide range of evolutionary computation studies. It is well known that the average height of local optima regresses to the mean of the landscape with increasing epistasis, $K$. This fact has been confirmed using both theoretical studies of landscape structure and empirical studies of evolutionary search. We show that the global optimum behaves quite differently: the expected value of the global maximum is highest in the maximally rugged case. Furthermore, we demonstrate that this expected value increases with $K$, despite the fact that the average fitness of the local optima decreases. That is, the highest peaks are found in the most rugged landscapes, scattered amongst masses of low-lying peaks. We find the asymptotic value of the global optimum as $N$ approaches infinity for both the smooth and maximally rugged cases. In evolutionary search, the optima that are found reflect the local optima that exist in the landscape, the size of these optima - which corresponds to the size of their basins of attraction, and the effort expended in the search process. Increasing the level of epistasis in an NK landscape stochastically introduces higher peaks, but renders them exponentially more difficult to find.
\end{abstract}

Categories and Subject Descriptors: I.6.5 [Model Development]: Modeling Methodologies

General Terms: Experimentation.

Keywords: NK landscapes, rugged landscapes, combinatorial landscapes, search, optimisation.

Permission to make digital or hard copies of all or part of this work for personal or classroom use is granted without fee provided that copies are not made or distributed for profit or commercial advantage and that copies bear this notice and the full citation on the first page. To copy otherwise, to republish, to post on servers or to redistribute to lists, requires prior specific permission and/or a fee.

GECCO'05, June 25-29, 2005, Washington, DC, USA.

Copyright 2005 ACM 1-59593-010-8/05/0006 ...\$5.00.

\section{THE NK FITNESS LANDSCAPE}

The NK landscape model was designed to develop intuitions about the structure of complex combinatorial fitness landscapes [7,9]. Over the last fifteen years, the NK model has become an important tool for modelling biological systems that incorporate combinatorial interactions, such as the evolution of gene networks [8] and the development of the immune system [10]. The NK landscape has also been widely used by the evolutionary computation community as a generator of epistatic landscapes that serve as important test functions for search and optimisation techniques $[4,6,14]$.

An NK system is defined by two parameters, $N$, the number of components and $K$, the number of epistatic interactions between components. Each component has a binary value so an NK system can be represented by a bit string of length $N$ in which the bit at locus $i$ marks the value of component $i$. Each locus makes a contribution to the total fitness of the system based on $K+1$ values: its own and those of the $K$ components to which it is linked. Each of the $2^{K+1}$ possible combinations of component values is mapped to an independent, uniformly distributed fitness contribution in the range $[0,1]$ (Figure 1). The total fitness of a system is the average of the $N$ fitness contributions. Epistatic interactions can either be allowed between random loci, or, as is this case in this study, restricted to adjacent loci.

Fitness landscapes can be explored through both mathematical analysis and empirical simulation. One of the simplest empirical techniques used to explore landscapes is an adaptive walk [9]. A large number of adaptive walks performed on a single landscape gives an estimate of the number of local optima in the landscape as well as their fitness values and the probability of reaching them from an arbitrary starting point. The set of starting points from which a given optimum can be reached is known as its basin of attraction. In general, a larger basin of attraction makes an optimum easier to find. Because certain optima may have larger basins of attraction than others, adaptive walks give a biased description of the optima in a landscape and are not 


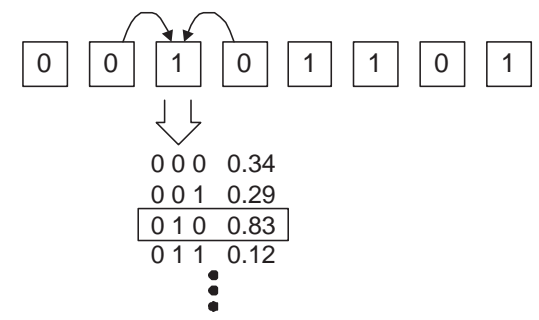

Figure 1: An NK system with $N=8$ and $K=2$. Epistatic interactions are shown for the third locus, whose fitness contribution is 0.83 . The boundary of the bit string representation is periodic; that is, the leftmost and rightmost loci are neighbours.

always guaranteed to find the global optimum. Note that, throughout this paper, the convention is that the optima of interest are maxima, rather than minima. The methods used apply to minima with only trivial modifications.

Landscapes generated using the NK model vary in size according to $N$ and in correlation according to $K$. When $K=0$ there are no interactions between loci and the resulting fitness landscape is smooth, containing only a single optimum. In this case all points in the landscape lie in the basin of attraction of the global optimum, making it trivially easy to find. As the number of interactions between loci increases, the number of local optima increases and the resulting landscape becomes more 'rugged'. The basin of attraction of each optimum (including the global optimum) decreases in size and the landscape becomes increasingly difficult to search. When the landscape is 'maximally rugged' (i.e., when $K=N-1$ ), the expected number of optima is $O\left(2^{N} / N\right)$ [17]. Any given starting point will be in the basin of attraction of only a small fraction of the optima, and only a small fraction of starting points will be in the basin of attraction of the global optimum.

When $K>0$, it is not possible to maximise the fitness contributions of all loci simultaneously as changes at any locus will also affect the fitness contributions of $K$ others. The fitness of the global maximum is therefore typically less than the sum of the maximum possible fitness of each locus. Weinberger [17] shows that, with increasing $K$, the fitness values of the many local optima asymptotically approach a normal distribution with a mean that regresses towards the landscape average. Previous research suggests that adaptive walks attain their highest fitness values on landscapes with a low level of ruggedness (around $K=2$ ) [8,17].

While the behaviour of the average local optima and the performance of adaptive walks are well-understood, many properties of NK landscapes remain unexplored. In this paper, we consider two in particular: the distribution of fitness values across the entire landscape, and the behaviour of the global optimum as a function of $N$ and $K$.

Weinberger [16] concluded that the fitness of the global optimum stays roughly constant as $N$ becomes large and gave bounds for the fitness of the global maximum in the case of an uncorrelated $(K=N-1)$ landscape. More recently, empirical studies $[12,13]$ propose that the global minimum decreases as $N$ increases (for $N$ in the range 10 to 25) and also as ruggedness increases. Other mathematical analyses of the global optima $[2,3,11]$ have employed non-uniform fitness contributions in order to improve ana- lytic tractability. These studies provide a number of useful results but are not directly applicable to the typical case of uniformly distributed fitness contributions used in most applications of the NK landscape (e.g. $[4,8]$ ).

In this study mathematical analysis and empirical simulation were used to investigate the case where fitness contributions are uniformly distributed on $[0,1]$. In Section 2 the distribution of fitness values is interpreted using the central limit theorem. To clearly illustrate how an actual landscape varies as $N$ gets large, the behaviour of the global extrema, which indicate the range of values in the landscape, is investigated. The asymptotic value of the global optimum of a maximally rugged landscape as $N \rightarrow \infty$ is then established under the Gaussian approximation. The behaviour of the global optimum has important implications for search, and Section 3 reports the results of simulations investigating the performance of adaptive walks as both number of searches and ruggedness of the landscape increase.

\section{LANDSCAPE STRUCTURE}

\subsection{Fitness distribution}

In an NK landscape, the fitness of a point, $W$, is the average of $N$ fitness contributions, $w_{i}$, each independently distributed as $U(0,1)$ random variables,

$$
W=\frac{1}{N} \sum_{i=1}^{N} w_{i} .
$$

By the central limit theorem, the fitness of a point, $W$, converges in distribution to a standard Gaussian random variable when appropriately scaled:

$$
\frac{W-\frac{1}{2}}{\sqrt{\frac{1}{12 N}}} \stackrel{D}{\longrightarrow} \operatorname{Normal}(0,1) \quad \text { as } \quad N \rightarrow \infty .
$$

For suitably large values of $N$, we can therefore approximate the fitness of any single point on the landscape using a random variable with a Gaussian distribution, with mean $\frac{1}{2}$ and variance $\frac{1}{12 N}$. This property holds for all $K$ - the level of epistasis is not relevant when considering the fitness of a single point, ignoring the rest of the landscape. However, epistatic interactions between loci are a critical feature of NK models, because they induce correlations between the fitnesses of different points on a given landscape.

The structure of the landscape is such that any two points on the landscape will share fitness contributions if they have identical values in a set of epistatically linked components. Because such points share some fitness contributions, and all other fitness contributions are mutually independent, the fitness values of these points will be positively correlated. When $K$ is low, each possible fitness contribution appears in a large proportion of the points in a landscape but as $K$ increases, the number of correlated points decreases. When $K=N-1$, no points have any fitness contributions in common, so that the landscape consists of $2^{N}$ independent random variables.

When $K=N-1$, the empirical fitness distribution of a landscape approaches a Gaussian distribution with mean $\frac{1}{2}$ and variance $\frac{1}{12 N}$. However, if $K<N-1$ then positive correlations between points on a specific landscape will cause its empirical fitness distribution to differ from the Gaussian approximation. Because the variance is proportional to $\frac{1}{N}$, 


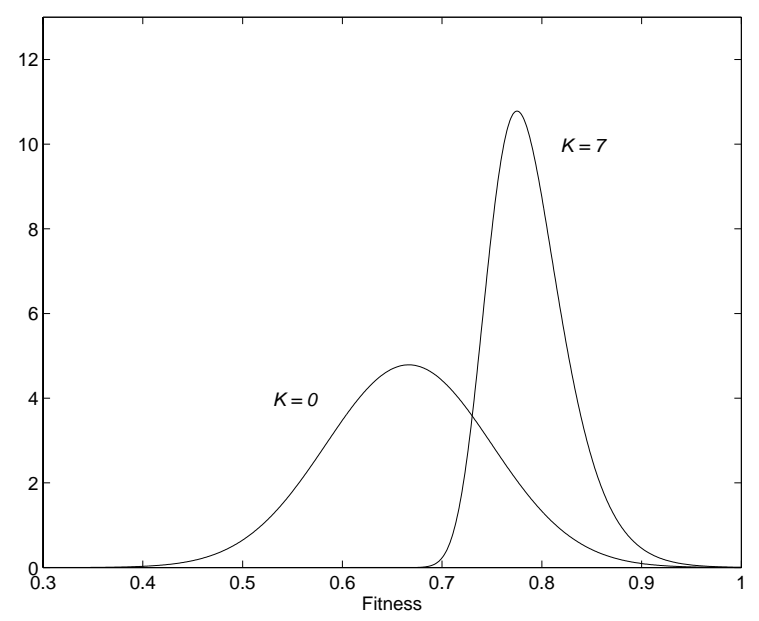

Figure 2: Gaussian approximations to the probability density functions of the value of the global optimum for $N=8$, with $K=7$ and $K=0$, using (2) and (4) respectively. The increased mean (and decreased variance) of the global maximum for $K=N-1$ over $K=0$ is clearly visible.

then as $N$ increases, the variance of a fitness value decreases and the landscape becomes flatter as values are clustered more closely around the mean value of $\frac{1}{2}$. The behaviour of the global extrema indicate how the range of fitness values decreases as $K$ decreases from $N-1$ (Figure 2).

\subsection{Behaviour of the global optimum}

Suppose that $W_{\max }(K)$ is the global maximum ${ }^{1}$ for an NK landscape with a level of epistasis, $K$. For $K=N-$ 1 , the global maximum is the largest of $2^{N}$ independent random quantities, each a sum of $N$ independent uniform random variables, and we will denote it by $W_{\max }(N-1)$. The probability density function of $W_{\max }(N-1)$ is

$$
f_{W_{\max }(N-1)}(z)=2^{N} f_{W}(z)\left(F_{W}(z)\right)^{2^{N}-1},
$$

where $f_{W}(z)$ is the probability density function of the fitness of a point, $W$, and $F_{W}(z)$ is the cumulative distribution function of $W$.

Conversely, for $K=0$ each locus has only 2 possible values (0 or 1), so the global maximum is the average of $N$ random variables, each of which is chosen as the largest of two independent samples from $U(0,1)$. The maximum and minimum of each pair have means of $\frac{2}{3}$ and $\frac{1}{3}$, respectively, and a common variance of $\frac{1}{18}$. Since each of the $N$ maxima (one from each pair) are independent, we can again use the central limit theorem to see that the global maximum for $K=0$ converges in distribution as

$$
\frac{W_{\max }(0)-\frac{2}{3}}{\sqrt{\frac{1}{18 N}}} \stackrel{D}{\longrightarrow} \operatorname{Normal}(0,1) .
$$

The preceding equations can be used to generate approximations to the probability distributions of the value of the global optimum when $K=0$ and $K=N-1$ (Figure 2).

\footnotetext{
${ }^{1}$ We focus on the behaviour of the global maximum with complementary results holding for the global minimum due to the symmetry of the fitness distribution.
}

Comparisons of the probability distributions of the global optima suggest that the mean of $W_{\max }(N-1)$ is greater than the mean of $W_{\max }(0)$ when fitness contributions are uniform on $[0,1]$. Suppose that rather than being uniform, fitness contributions have an arbitrary distribution. Then, we can show (Appendix A) that larger global maxima are more likely to occur in landscapes where $K=N-1$ than in landscapes where $K<N-1$. Equivalently, we say that the global maximum for a landscape with $K=N-1$ is strictly stochastically greater than the global maximum of any landscape with $K<N-1$ :

$$
P\left[W_{\max }(J)>z\right]<P\left[W_{\max }(N-1)>z\right] .
$$

where $J<N-1$ and $z \in[0,1]$.

Stochastic ordering implies ordering of expectation, so it is a direct consequence of these results that the expected value of the global maximum is largest in the maximally rugged case:

$$
\mathrm{E}\left[W_{\max }(J)\right]<\mathrm{E}\left[W_{\max }(N-1)\right],
$$

where these expectations exist.

We conjecture that the global maximum is stochastically non-decreasing in $K$ for fixed $N$ :

$$
P\left[W_{\max }\left(J_{1}\right)>z\right] \leq P\left[W_{\max }\left(J_{2}\right)>z\right], \quad J_{1}<J_{2} .
$$

Our conjecture suggests that there is a hierarchy of distributions of global maxima, corresponding to the level of epistatic interaction, $K$ : the greater the value of $K$, the greater the global maximum is likely to be. Proving (7) is difficult in the case of arbitrary fitness contributions. However, Evans and Steinsaltz [3] establish that regardless of the distribution of the fitness values, in the limit as $N$ goes to infinity the asymptotic global maximum is non-decreasing in $K$; that is, if $J_{1}<J_{2}$,

$$
\lim _{N \rightarrow \infty} W_{\max }\left(J_{1}\right) \leq \lim _{N \rightarrow \infty} W_{\max }\left(J_{2}\right),
$$

which is consistent with the above conjecture. However, when $N$ is finite and excluding the case where fitness contributions are Gaussian it remains necessary to use empirical investigation. Computer simulations of the uniform case indicate that the global maximum is indeed stochastically increasing in $K$ (Figure 3 ).

\subsection{Asymptotic value of the global extrema}

Here we derive the limiting value of the mode of the global maximum, as $N \rightarrow \infty$ and $K=N-1$. Asymptotics of the height of the global maximum have been considered by other authors; Durrett and Limic [2] derive central limit theorems for the height of the global maximum, and give a bound for this height for all values of $K$ in the limit as $N \rightarrow \infty$, in the case where fitness contributions are the negatives of exponential random variables. Evans and Steinsaltz [3] derive the limiting value of the global maximum for $K=1$ in the case where the fitness contributions have the exponential distribution, but more importantly, also demonstrate that such limiting values exist for all fixed $K$ for general distributions of fitness contributions. Here, we address the situation where $W$ obeys the Gaussian approximation for uniformly distributed fitness contributions.

Following Weinberger [16] we can establish that when the Gaussian approximation (2) is valid and $K=N-1$, the globally maximal fitness must lie between $\frac{1}{2}+\sqrt{\frac{\ln 2}{6}}$ and 


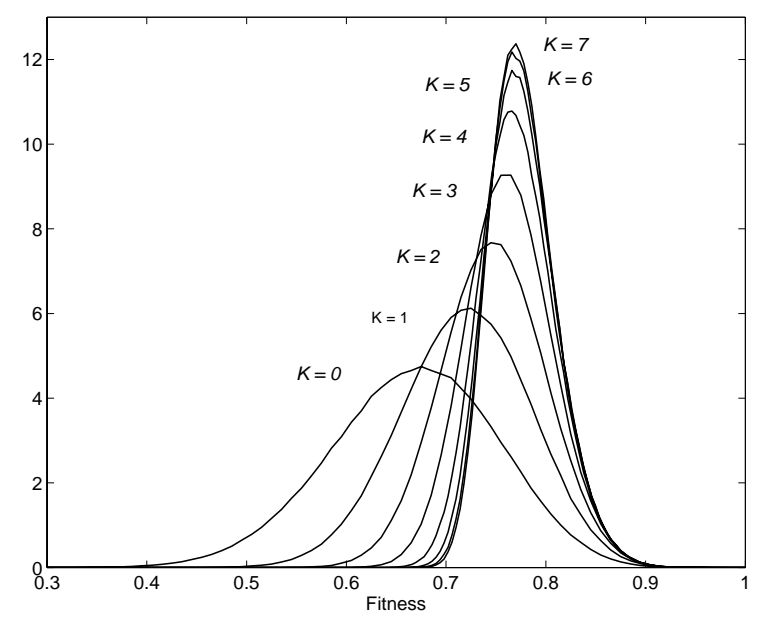

Figure 3: Empirical probability density functions for the global optimum for $N=8$, averaged over one million landscapes for each value of $K$. The expected global optimum increases as $K$ increases.

$\frac{1}{2}+\sqrt{\frac{\ln 2}{3}}$. It is known that the global maximum converges in probability to a constant for fixed $K$ [3], and hence that the variance of the global maximum goes to zero as $N$ becomes large. We determine a specific value for the peak of the distribution of the global optimum as $N \rightarrow \infty$.

The mode of the global optimum is the critical point, $z^{*}$, of the distribution function given by $(3)$ in the range $[0,1]$. Since $W$ converges in distribution to the constant value of $\frac{1}{2}$ as $N \rightarrow \infty, \lim _{N \rightarrow \infty} F_{W}(z)=1$ for $z \geq \frac{1}{2}$, and so (3) can be solved to find

$$
z^{*}=\frac{1}{2}+\sqrt{\frac{1}{12 N} \Omega\left(\frac{\left(2^{N}-1\right)^{2}}{2 \pi}\right)},
$$

for any $N$, where $\Omega$ is Lambert's W function (see Appendix B). Now, $\Omega(x) \sim \ln (x)$ as $x \rightarrow \infty$, so

$$
\lim _{N \rightarrow \infty} z^{*}=\frac{1}{2}+\sqrt{\frac{\ln 2}{6}} \approx 0.84 .
$$

As $N \rightarrow \infty$ the variance of the distribution $f_{W_{\max }(N-1)}$ decreases while the peak of the distribution approaches $\frac{1}{2}+\sqrt{\frac{\ln 2}{6}}$, so that in the maximally rugged case the most likely value of the global optimum approaches 0.84 as $N$ gets large. We note, in particular, that this corresponds to the lower bound derived by Weinberger [16].

\section{IMPLICATIONS FOR SEARCH}

NK landscapes have been used both as a model of evolutionary search and as test functions for computational search and optimisation techniques. Here we examine some implications of the results from Section 2 for the performance of search algorithms. One of the simplest empirical techniques used to explore landscapes is an adaptive walk, performed by a random mutation hill-climbing algorithm. This algorithm starts at a randomly chosen point and proceeds until an optimum is found. At each step of the algorithm, the value of a randomly chosen component is altered; if the change results in a fitness increase it is retained, otherwise it is discarded. The sequence of increasingly fitter points followed

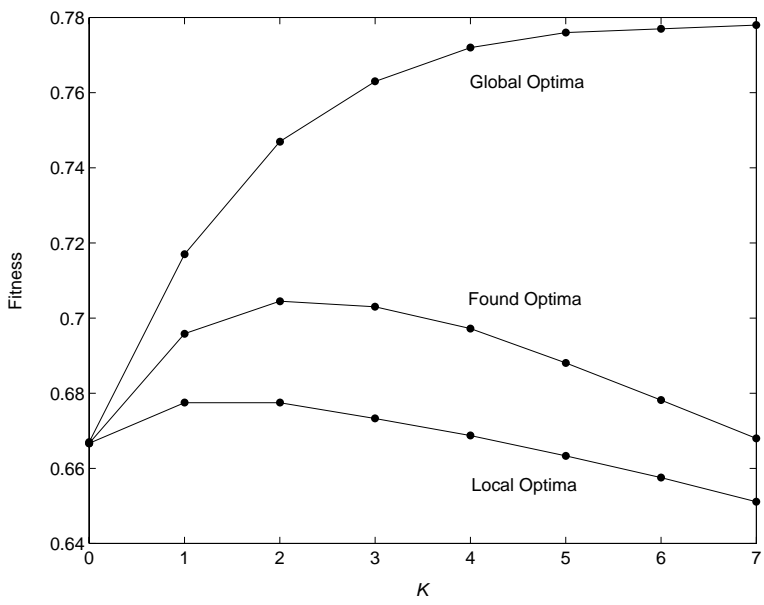

Figure 4: The fitness of the global, local and found optima averaged over 1000 landscapes with $N=8$. As $K$ increases, the global optimum increases while the average fitness of the local optima decreases. The average optima actually found by hill-climbing tend to be greater than the average of all local optima, because higher local optima tend to have larger basins of attraction. For $N=8$, the fittest optima are found when $K=2$.

by a hill-climber is known as an adaptive walk. This search algorithm corresponds in principle to a minimal evolutionary algorithm: the $(1+1)$ ES [1].

Computer simulations were used to obtain empirical values for the average heights of the local optima, the global optimum, and the optimum found by a single hill-climber, on landscapes with $N=8$ (Figure 4 ). These values were calculated by first constructing 1000 landscapes then computing averages of local and global optima as well as the performance of all possible hill-climbers from all possible starting points. As conjectured in Section 2, the expected height of the global optimum increases with $K$, and this value is largest when $K=N-1$. However, the average height of the local optima decreases after $K=1$, and the average height of the optimum found by a single hill-climber decreases after $K=2$, in agreement with previous studies $[8,17]$.

Furthermore, the average of the found optima is consistently larger than the average of the local optima. Given that a hill-climber starts at a random point, the tendency to reach higher-than-average optima suggests that these optima have larger basins of attraction, a feature of NK landscapes observed by Kauffman [8]. For $K=0$ there is only one optimum with a basin of size $2^{N}$, but as $K$ increases, the number of optima increases, so that the average size of the basins of attraction must decrease. Thus, while the expected fitness of the global optimum increases with the level of epistatic interaction, local optima become more numerous with a lower average fitness, so that the optimum found by a single hill-climber also tends to be lower.

Since a single hill-climber can only reach a limited number of optima in a rugged landscape, a population of hill-climbers is able to explore more of the landscape and find fitter optima (Figure 5). Increasing the number of hill-climbers does result in fitter optima being found, however the relative ad- 


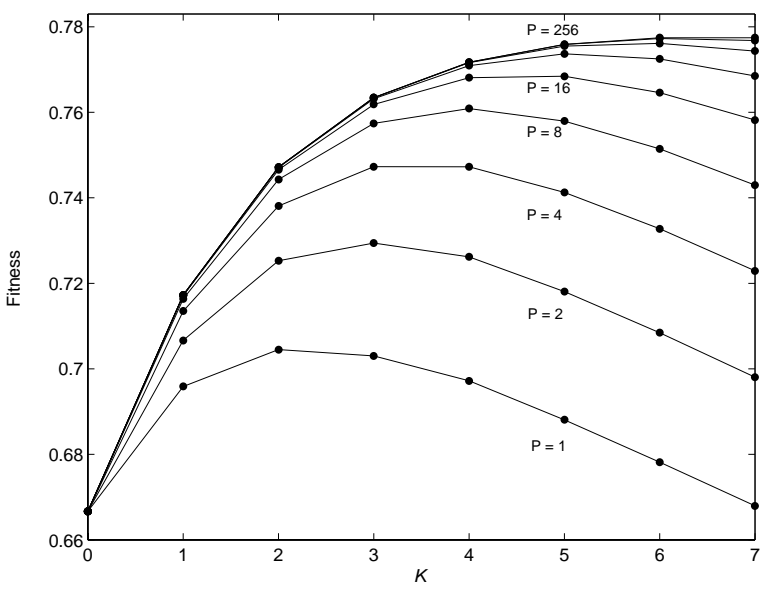

Figure 5: The best fitness from a population of $P$ hill-climbers averaged over 1000 landscapes with $N=8$. As populations increase in size, the average best fitness found occurs in increasingly rugged landscapes.

vantage of doubling the population size diminishes as the population becomes larger. The diminishing return from extra hill-climbers is due to the redundancy of an increasing number of hill-climbers starting in the same basin of attraction and hence reaching the same local optima. When $K=N-1$, the number of hill-climbers required to find the global optimum approaches $2^{N}$, equal to the size of the landscape.

\section{CONCLUSION}

When using a model such as the NK landscape in practical situations, it is important to understand the expected properties of landscapes so that results can be related to known quantities. In this study, questions about these properties were addressed from two perspectives: the structure of the landscapes, in particular the behaviour of the global optimum, and the ability of adaptive walks to find the global optimum.

It is well known that the height of optima found by evolutionary search on NK landscapes decreases with increasing $K$. However this fact does not reflect the behaviour of the global optimum which achieves its highest value when $K=N-1$.

This study considered NK landscapes with uniform fitness contributions: the central limit theorem was used to determine an approximate distribution for the landscape, and the effect of $N$ and $K$ on this approximation was identified (Section 2). It is conjectured that the global maximum is stochastically non-decreasing in $K$ and it has been proved that the expected value of the global maximum is largest when $K=N-1$. The asymptotic value of the global optima was found for $K=N-1$ as $N \rightarrow \infty$.

Search performance on landscapes with varying degrees of ruggedness was investigated (Section 3). It was found that, while it is possible for an adaptive walk to find the global optima on increasingly rugged landscapes, the effort required to find them increases dramatically.

It is well-known that increasing the number of epistatic interactions creates landscapes with many more peaks of lower average height, and that the greater complexity of these landscapes makes search increasingly difficult. The results in this paper add the additional insight that the very highest peaks may be found in the most rugged landscapes, scattered among the masses of low lying peaks. Any increase in the level of epistasis has the potential to introduce higher peaks, but simultaneously renders these peaks increasingly difficult to find.

\section{Acknowledgements}

This study was supported by an Australian Postgraduate Award to NG and an Australian Research Council (ARC) grant to JW. The work of $\mathrm{BC}$ is supported by a $\mathrm{PhD}$ scholarship from the ARC Centre of Excellence for Mathematics and Statistics of Complex Systems. BT was hosted by the School of ITEE, UQ when this study was carried out.

\section{REFERENCES}

[1] T. Bäck, D. Fogel, and Z. Michalewicz. Handbook of Evolutionary Computation. Oxford University Press, Oxford, UK, 1997.

[2] R. Durrett and V. Limic. Rigorous results for the NK model. The Annals of Probability, 31:1713-1753, 2003.

[3] S. N. Evans and D. Steinsaltz. Estimating some features of NK fitness landscapes. Annals of Applied Probability, 12(4):1299-1321, 2002.

[4] R. B. Heckendorn, S. Rana, and D. L. Whitley. Test function generators as embedded landscapes. In W. Banzhaf and C. Reeves, editors, Foundations of Genetic Algorithms 5, pages 183-198. Morgan Kaufmann, San Francisco, CA, 1999.

[5] F. P. J. D. Esary and D. W. Walkup. Association of random variables, with applications. Annals of Mathematical Statistics, 38(5):1466-1474, 1967.

[6] K. A. D. Jong, M. A. Potter, and W. M. Spears. Using problem generators to explore the effects of epistasis. In T. Bäck, editor, Proceedings of the Seventh International Conference on Genetic Algorithms (ICGA97), San Francisco, CA, 1997. Morgan Kaufmann.

[7] S. A. Kauffman. Adaptation on rugged fitness landscapes. In D. Stein, editor, Lectures in the Sciences of Complexity, volume 1 of SFI Studies in the Sciences of Complexity, pages 527-618. Addison-Wesley, Redwood City, 1989.

[8] S. A. Kauffman. The Origins of Order: Self-Organization and Selection in Evolution. Oxford University Press, 1993.

[9] S. A. Kauffman and S. Levin. Towards a general theory of adaptive walks on rugged landscapes. Journal of Theoretical Biology, 128:11-45, 1987.

[10] S. A. Kauffman and E. D. Weinberger. The NK model of rugged fitness landscapes and its application to maturation of the immune response. Journal of Theoretical Biology, 141:211-245, 1989.

[11] V. Limic and R. Pemantle. More rigorous results for the NK model. http://arxiv.org/abs/math/0308282, 2003.

[12] K. E. Mathias, L. J. Eshelman, and J. D. Schaffer. Niches in NK-landscapes. In Foundations of Genetic Algorithms (FOGA-6), pages 27-46. Morgan Kauffman, 2001. 
[13] R. E. Smith and J. E. Smith. An examination of tunable, random search landscapes. In Foundations Genetic Algorithms (FOGA-5), pages 165-177. Morgan Kauffman, 1999.

[14] R. E. Smith and J. E. Smith. New methods for tunable, random landscapes. In W. N. Martin and W. M. Spears, editors, Foundations of Genetic Algorithms 6. Morgan Kaufmann, 2001.

[15] Y. L. Tong. Probability Inequalities in Multivariate Distributions. Probability and Mathematical Statistics. Academic Press, New York, 1980.

[16] E. Weinberger. Correlated and uncorrelated fitness landscapes and how to tell the difference. Biological Cybernetics, 63:325-336, 1990.

[17] E. D. Weinberger. Local Properties of Kauffman's N-k model: A Tunably Rugged Energy Landscape. Physical Review A, 44(10):6399-6413, 1991.

\section{APPENDIX}

\section{A. THE DISTRIBUTION OF $W_{\max }(K)$}

The derivation of (5) relies on the concept of association, which encompasses a particular notion of positive dependence between random variables. The elements, $X_{1}, \ldots, X_{n}$, of a random vector, $\mathbf{X}$, are said to be associated if

$$
\operatorname{Cov}\left[g_{1}(\mathbf{X}), g_{2}(\mathbf{X})\right] \geq 0
$$

where this quantity exists, for all functions $g_{1}$ and $g_{2}$ monotonically non-decreasing in each of the $n$ elements of $\mathbf{X}$. Independent random variables are associated.

Important properties of associated random variables include the following (see [5] and [15] for further details):

1. Suppose there is a sequence

$$
g_{i}=g_{i}\left(X_{1}, X_{2}, \ldots, X_{n}\right), \quad i=1, \ldots, m .
$$

where each $g_{i}(\cdot)$ is a non-decreasing function of its arguments. If $\mathbf{X}$ is a vector of associated random variables, then the $g_{i}$ are also associated.

2. If the elements of $\mathbf{X}, X_{1}, \ldots, X_{n}$, are associated random variables,

$$
P\left[\bigcap_{i=1}^{n}\left\{X_{i} \leq z_{i}\right\}\right] \geq \prod_{i=1}^{n} P\left[X_{i} \leq z_{i}\right]
$$

for some vector of values $\mathbf{z}$.

In order to utilise the properites of association, the fitness landscape can be constructed as a $2^{N}$-element vector, $\mathbf{W}_{K}$. It is consistent with the NK model that these values may be generated from an $N 2^{N}$-element vector of fitness components, f, by multiplying f by a $2^{N} \times N 2^{N}$ matrix of zeros and ones, $M_{K}$, and dividing by $N$. The elements of $M_{K}$ indicate which elements of $\mathbf{f}$ contribute to which fitnesses (when $K<N-1$, some elements of $\mathbf{f}$ will not contribute to any fitnesses). Hence, recalling (1), $\mathbf{W}_{K}$ is a function of $\mathbf{f}$ :

$$
\mathbf{W}_{K}(\mathbf{f})=\frac{1}{N} M_{K} \mathbf{f}
$$

Because the $f_{i}$ are all independent, $\mathbf{f}$ is a vector of associated random variables. Thus, since the rows of $M_{K} / N$ form a sequence of nondecreasing functions of $\mathbf{f}, \mathbf{W}_{K}(\mathbf{f})$ is also a vector of associated random variables (by Property 1, above).

Note that the event that the maximum of the fitnesses is less than some value, $z$, is the same as the event that all the fitnesses are less than $z$. That is, for any $J \leq N-1$, and if $W_{1}, \ldots, W_{2^{N}}$ are the elements of $\mathbf{W}_{K}$,

$$
P\left[W_{\max }(J) \leq z\right]=P\left[\bigcap_{i=1}^{2^{N}}\left\{W_{i} \leq z\right\}\right]
$$

Consequently, from (11) and (13), the global maxima for all $J \leq N-1$ satisfy

$$
P\left[W_{\max }(J) \leq z\right] \geq \prod_{i=1}^{2^{N}} P\left[W_{i} \leq z\right]
$$

where there is equality only when $J=N-1$, in which case the fitness of each point in the landscape must be independent. Hence, the maximum of the fitnesses for $K=N-1$, $W_{\max }(N-1)$, is stochastically greater than $W_{\max }(J)$, for any $J<N-1$, as stated by (5).

\section{B. ASYMPTOTIC BEHAVIOUR OF THE GLOBAL MAXIMUM}

The most likely value of the global optimum is the peak of the probability density function $f_{W_{\max }(N-1)}(z)$. Hence, the most likely value is $z=z^{*}$ such that

$$
\frac{d}{d z} f_{W_{\max }(N-1)}(z)=0 .
$$

For finite $z$, this becomes

$$
12 N\left(z^{*}-\frac{1}{2}\right) F_{W}\left(z^{*}\right)=\left(2^{N}-1\right) f_{W}\left(z^{*}\right),
$$

and in this case the fitness $W$ has a normal distribution with mean $\frac{1}{2}$ and variance $1 / 12 N$ (these parameters come from the normal approximation for fitness contributions uniformly distributed between 0 and 1). Thus

$$
12 N\left(z^{*}-\frac{1}{2}\right) F_{W}\left(z^{*}\right)=\left(2^{N}-1\right) \sqrt{\frac{12 N}{2 \pi}} e^{-6 N\left(z^{*}-\frac{1}{2}\right)^{2}} .
$$

Rearranging and squaring both sides,

$$
12 N\left(z^{*}-\frac{1}{2}\right)^{2} e^{12 N\left(z^{*}-\frac{1}{2}\right)^{2}}=\left(\frac{2^{N}-1}{F_{W}\left(z^{*}\right) \sqrt{2 \pi}}\right)^{2},
$$

which is of the form $A e^{A}=B$, and has the solution $A=$ $\Omega(B)$ where $\Omega$ is Lambert's W-function. Hence, $z^{*}$ satisfies

$$
12 N\left(z^{*}-\frac{1}{2}\right)^{2}=\Omega\left(\frac{\left(2^{N}-1\right)^{2}}{2 \pi F_{W}\left(z^{*}\right)^{2}}\right) .
$$

The argument of $\Omega$ in this expression goes to infinity as $N$ goes to infinity, but $\Omega(x) \sim \ln (x)$ as $x \rightarrow \infty$. Then, from (5), $z^{*} \geq \frac{1}{2}$ and so $F_{W}\left(z^{*}\right) \rightarrow 1$ as $N \rightarrow \infty$, and so

$$
\left(z^{*}-\frac{1}{2}\right)^{2} \sim \frac{\ln 2}{6}
$$

from which (8) follows. 